\section{UNDERSTAND AND PREPARE FOR EBOLA}

As infection control professionals will be on the front line to cope with the Ebola outbreak if it reaches UK hospitals, Bioquell has collated a range of useful resources to help them understand and prepare for the Ebola challenge as highlighted by the World Health Organisation.

Bioquell's Ebola resources and information webpage, www.bioquell.com/ebola-resources, provides a range of useful information about the Ebola virus, infection prevention and control as well as specific decontamination considerations. Their expert microbiology team has put together two case studies which highlight how Bioquell's hydrogen peroxide vapour technology has successfully been used in the past to eradicate infectious diseases similar to Ebola. A white paper is also available to explain how Bioquell's solutions (HPV equipment and services and the Bioquell Pod) are effective and fast ways to put the UK Department of Health's guidelines into practice in the fight against Ebola.

Visit Bioquell's Ebola resources and information webpage to find out more at www.bioquell.com/ ebola-resources.

\section{REWARDING INFECTION CONTROL}

Purchasing infection control products has never been more rewarding thanks to DENTSPLY Rewards, the scheme that offers you the opportunity to earn 'Rewards £s' every time you order from the DENTSPLY Sultan Healthcare range.

Specifically designed to help meet the essential requirements of HTM 01-05, DENTSPLY Sultan Healthcare delivers a range of high quality, value-driven infection prevention products. Their personal preventive equipment solutions, such as Com-Fit masks, feature excellent breathability and high filtration, which helps protect users against blood and body fluids. The market-leading ASSURE Plus self-sealing sterilisation pouches confirm heat and gas penetration ensuring complete protection of all your valuable surgery instruments. DENTSPLY Sultan Healthcare's disposable 3 in 1 syringe tip - FlashTip - presents a low cost solution to help minimise cross infection.

DENTSPLY Rewards is a secure and simple way to purchase these popular, value for money products, available online, 24 hours a day. You can now maintain protection for both you and your patients, comply with HTM 01-05 and earn fantastic 'DENTSPLY Rewards £s' at the same time.

www.dentsplyrewards.co.uk.

\section{AVOID THE YUCK FACTOR}

Bib-Eze Hygienic Bib Holders from DUX Dental are the ideal way to minimise the risks of cross-contamination in your dental surgery and avoid the 'yuck factor' of reusable bib clips.

There is a substantial body of evidence forming that suggests disposable bib holders represent the safest way for clinicians to avoid cross-contamination from reusable bib clips and chains. To eliminate this threat DUX Dental has designed disposable Bib-Eze Hygienic Bib Holders. Created for optimal hygiene, you can now be assured that the bib holder you use does not carry bacteria, hair or sweat from a previous patient.

One size fits all as the bib holder, made using soft crepe paper, stretches to fit the patient. Bib-Eze are comfortable and can be used in combination with any paper bib, making them the perfect bib holder for any practice.

Maximise your standards of infection control today with Bib-Eze Hygienic Bib Holders from DUX Dental.

wWw. dux-dental.com

\section{COST-EFFECTIVE, ECO-FRIENDLY CLOTHS}

Unlike conventional cloths, Evident's range of reusable, washable microfibre cloths, e-cloth Professional, have 30,000 fibres per square inch and more than 500 million fibre strands per cloth. These special fibres are capable of entangling and thus remove a wide range of pathogenic particles. When damp, its capillary action rapidly draws

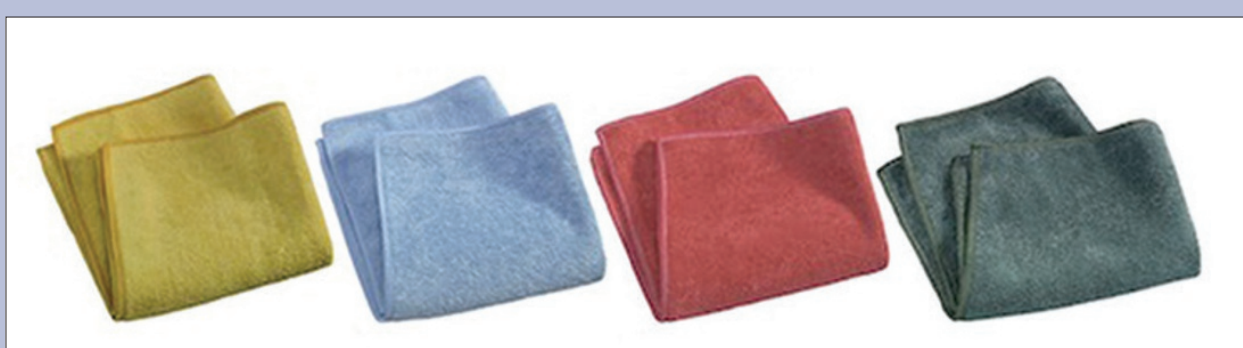

contamination into the fibre gaps, making it effective in removing grease, dirt and bacteria from surfaces, which other cloths leave behind. In addition, every individual cloth is impregnated with antimicrobial nanosilver, which remains active throughout the life of the cloth.

(1)

